\title{
Building Detection in High Resolution Remotely Sensed Images based on Automatic Histogram-Based Fuzzy C-Means Algorithm
}

\author{
Dong Jo Kim1), P. Lakshmi Manjusha2)
}

\begin{abstract}
Building detection is a fundamental and challenging task in satellite image analysis and has significant importance in the wide range of applications such as creation and update of maps and GIS database, urban monitoring, and planning application. This paper presents an approach for building detection in high resolution remotely sensed images. The proposed approach firstly segmented the pan-sharpened image by Automatic Histogram based Fuzzy C-Means (AHFCM) algorithm. Secondly the vegetation and shadow regions are eliminated by using Normalized Difference Vegetation Index (NDVI) and ratio map in HIS model respectively. Finally the buildings and non building regions are classified by using Principal Component Analysis (PCA) and outcomes are further refined by morphological operations.
\end{abstract}

Keywords : building detection, NDVI, PCA, AHFCM, fuzzy, histogram, GIS.

\section{Introduction}

The automation has been part of day to day life in present era of developed technologies. Detection of buildings from monocular aerial and satellite images has become an important issue to create and update maps and GIS database for the better performance in cases of change detection, land use analysis and urban monitoring applications. The traditional manual processing of the images is time consuming and expensive, which requires continuous and prolonged human intervention, hard work and attention[1-3]. The rapidly growing urban areas in the major cities has compelled and necessitated the automatic detection of buildings from

Received(December 29, 2016), Review Result(1st: January 16, 2017, 2nd: February 25, 2017), Accepted(March 10, 2017)

${ }^{1}$ Seoul Media Institute of Technology, 7F, 402 Worldcupbuk-ro, Mapo-gu, Seoul, Korea email: sojudj@gmail.com

${ }^{2}$ (Corresponding Author) Department of Computer Science and Engineering, KL University email: lakshmimanjusha16@gmail.com 
remote sensing images and is a very active field of research in present scenario. Extraction of man-made structures from remotely sensed images is difficult because images have a wide variety of structured and unstructured contents. Images may differ in resolution, sensor type, orientation, quality, dynamic range, illumination conditions, and seasons, etc. besides these reasons buildings may have complicated structures that may appear as indistinguishable from roads and pavements and also rooftops may reflect fragmented characteristics due to shading. They might be occluded by other buildings or vegetation[4-6]. In return for this, the advances in remote sensing enabled the availability of wide variety of data in the problem domain, which provide many features to be employed or to be fused if possible. As a result, due to the complexity and diversity of problem domain, it is hard to develop generic algorithms and methods for building extraction (i.e., detection and delineation of buildings) from numerous types of data. Therefore, the methodologies existing in the literature are mostly restricted to specific types of buildings and data. It seems to be promising that, with the availability of wide range of data feature level fusion incorporated into structural information the performance of manmade structure extraction would be improved. Besides, this would increase the generic characteristic of methodologies as well. In general, the problem of building extraction can be divided into low level and high level tasks. Initially, low level tasks concentrate on determining the region of interest. Then, high level tasks (feature extraction and classification) are performed. In the literature, different kinds of features are defined and the researches are mainly concentrated on forming feature spaces[7]. After the detection of features, these features are either classified if supervision is applicable or clustered if supervision is not applicable. Features which are widely used in the literature can be grouped as geometric, photometric and structural. Geometric features define basic geometrical properties such as area, circumference, roundness, right angles, comers, straight lines etc. Photometric features are related to color information. Structural features refer to connectedness of neighbors according to some similarity measures. Since the structures in an urban remote sensing image can be classified into several classes such as buildings, vegetation, roads and water areas, a further classification of the content using rule based and context driven approaches is necessary. In doing this, density (rural, suburban, urban), object complexity (residential, industrial, military), architecture (elaborate, plain, none), terrain defined by Digital Elevation Model (DEM) (flat, hilly, mountainous), or vegetation defined by NDVI (none, moderate, heavy) are taken into account. Some studies concentrate on extracting low level features only and subsequently set relations among them in favor of supporting a hypothesis of building[1-4]. However, although the approaches and algorithms range over a large extent regardless of data utilized and strategies 
to be adopted, it is important to utilize the high level information as completely as possible[8][9].

This paper presents a generic algorithm for automatic extraction of buildings from high resolution satellite images from urban environments. The paper is organized as follows: the proposed methodology is presented in Section II, experimental results in section III and conclusion with future scope in section IV.

\section{Building Detection}

\subsection{Methodology}

The proposed method relies on mainly three steps: first, segment the pansharpened image by automatic histogram based fuzzy c-means algorithm, secondly eliminate vegetation and shadow segment and finally filtering the thin, long and small artifacts by means of PCA and morphological thinning and opening. The main steps to be followed in the methodology are given as:

\section{A. Automatic Histogram-Based Fuzzy C-Means segmentation}

In order to cluster the image, the Fuzzy C-Means (FCM) clustering algorithm needs a value to be entered by a user as the number of clusters. Furthermore, the initial membership matrix is generated randomly in the FCM algorithm. The AHFCM[5-9] algorithm overcomes these limitations using image histograms and an image fusion technique. The AHFCM algorithm starts by composing the histogram of the brightness values (BVs) within the image for each band of images. Then, it computes the slopes between the point of the histogram under consideration and its previous and next points.

Let $\mathrm{x}_{\mathrm{q}}$ and $\mathrm{y}_{\mathrm{q}}$ are the coordinates (brightness and frequency values, respectively) in the histogram for the point $\mathrm{q}$, which we want to, decide whether it is a cluster center or not. Subsequently $\left(x_{q-1} ; y_{q-1}\right)$ and $\left(x_{q+1} ; y_{q+1}\right)$ are the coordinates of the previous and next points of the point $\mathrm{q}$ in the histogram, respectively.

Indeed, the above-mentioned rules remove the points which cannot be cluster centers. Thus, the remaining points are selected to be used in further processes. A thresholding process based on Euclidean distance is executed to merge the cluster centers which satisfy specified threshold value. Eventually, the BVs of the remaining points are the initial cluster centers and the 
number of cluster centers is the number of clusters for each band of the image. Using this initial information, any band of the image is labeled by executing the FCM algorithm.

The labeled images of the bands are fused using their labels to compute the initial cluster centers. Then numbers of clusters are automatically calculated for the multispectral image.

However, only unique numbers as labels for each pixel are needed to compute the number of cluster. Thus, the following equation is used to carry out the final unique labels.

Accordingly, initial cluster centers for the multispectral image are computed by selecting the cluster centers which correspond to their label values. Moreover, to get the appropriate clustering result, a thresholding process is used to restrict the distances between cluster centers as follows:

$$
\text { If Dki } \geq T 2, \text { then } C_{j d}{ }^{\prime} \text { is a cluster center, where Dki }=\left(C_{j d}{ }^{\prime}-C_{j d}{ }^{\prime}\right), i=(1 ; 2, \ldots \ldots . . j)
$$

The FCM is implemented to obtain the final clustered image of the multispectral image utilizing prior information (cluster centers and number of cluster).

\section{B. Pre-Processing: Elimination of Vegetation and Shadow Regions}

We identify and discard the segments which belong to vegetation and shadow regions to avoid the false alarms in building detection. Where $\mathrm{R}$ and NIR represent red and near-infrared bands, respectively. For each image, a threshold value tNDVI is computed using the Otsu method[3]. Then, the pixels whose NDVI values are greater than tNDVI are marked as vegetation pixels, and a vegetation mask is generated. The number of vegetation pixels in each segment si is defined as $M_{i}^{V}$. Then, a segment si is labeled as vegetation, if $M_{i}^{V}>\Lambda M_{i}$

Moreover, we use a multi-spectral false color shadow detection algorithm proposed by Teke et al. [4] due to two important reasons: (i) their proposed approach utilizes the advantage of the NIR band, and (ii) the shadow detection algorithm does not require user defined thresholds. In this approach, NIR, R and G are used to generate a false color image. Once the false color image (NIR-R-G) is obtained, it is converted into HSI color space.

Where $S$ is the normalized saturation, and $I$ is the normalized intensity. The extracted RatioMap is binarized using Otsu method to obtain a set of pixels which represents both shadows and vegetations. After the vegetation pixels are eliminated using NDVI, the segment $s_{i}$ is labeled as shadow, if $M_{i}^{s}>\Lambda M_{i}$, where $M_{i}^{s}$ is the number of shadow pixels in $\mathrm{s}_{\mathrm{i}}$. 


\section{PCA \& Morphological Filtering}

In order to decide on whether a given segment is an artifact or not, principle component analysis (PCA) [5] is applied to each segment in order to compute the extend of the segment in terms of principle components. The artifact whose area is smaller but not thin enough is eliminated by comparing their area to a threshold which is an empirical evidence of not being a building. The long artifacts such as roads are removed by thresholding the length and with ratio. Further the outcomes are refined by morphological operations.

\subsection{Experimental Results}

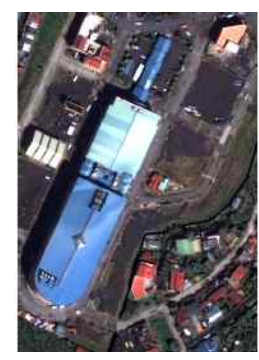

(a)

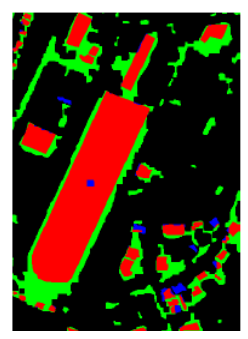

(b)

[Fig. 1] AHFCM Based Building Detection-(a) Input Image (b) Image showing output results as Red: TP, Blue: FN, Green: FP

The dataset used is a diverse collection of both manmade and natural objects and building characteristics ensuring very generic test case to evaluate the performance and robustness of the algorithm. Figure shows (a) the input pansharpened image and (b) its corresponding output. Red region shows the correctly extracted buildings.

\section{Conclusion}

The proposed algorithm provides automatic extraction of buildings from high resolution satellite images. Moreover, we are planning to improve the accuracy of the methodology by working on the automatic selection of threshold for AHFCM algorithm as well as implementing rule-based approaches. 


\section{References}

[1] C. J. Tucker, Red and photographic infrared linear combinations for monitoring vegetation, Remote Sens. Environ., (1979), Vol.8, No.2, pp.127-150.

[2] A. Katartzis and H. Sahli, A Stochastic Framework for the Identification of Building Rooftops Using a Single Remote Sensing Image, IEEE Transactions On GeoScience And Remote Sensing, (2008), Vol.46, No.1.

[3] N. Otsu, A threshold selection method from gray-level histograms, Automatica, (1975), Vol.11, No.285-296, pp.23-27.

[4] M. Teke, E. Baseski, A. O. Ok, B. Yuksel and C. Senaras, Multi-spectral false color shadow detection, in Proc. ISPRS Conf. Photogramm. Image Analysis (PIA'11), (2011), pp.109-119.

[5] Ö. Aytekin, İ. Ulusoy, A. Erener and H. Duzgun, Automatic and unsupervised building extraction in complex urban environments from multi spectral satellite imagery, in Proc. 4th Int. Conf. Recent Adv. Space Technol. (RAST'09), (2009), pp.287-291.

[6] S. Ghaffarian and S. Ghaffarian, Automatic histogram-based fuzzy C-means clustering for remote sensing imagery, ISPRS J. Photogramm. Remote Sens., (2014), Vol.97, pp.46-57.

[7] B. Zou, Y. Zhang, C. Wang, and Y. Cheng, Building cognition method based on human images cognition mechanism in high resolution PolSAR images, Geoscience and Remote Sensing Symposium (IGARSS), 2015 IEEE International, (2015) July 26-31; Milan, Italy.

[8] I. Khosravi, M. Momeni, and M. Rahnemoonfar, Performance Evaluation of Object-based and Pixel-based Building Detection Algorithms from Very High Spatial Resolution Imagery, Photogrammetric Engineering \& Remote Sensing, (2014), Vol.80, No.5, pp.519-528.

[9] M. D. Mura, J.A. Benediktsson, B. Waske, and L. Bruzzone, Morphological attribute profiles for the analysis of very highresolution images, IEEE Transactions on Geoscience and Remote Sensing, (2010), Vol.48, No.10, pp.3747-3762. 